\title{
The Effect of Posterior Pericardiotomy on Postoperative Pericardial Effusion in Coronary Artery Bypass Surgery
}

\author{
Mohamed A. Amr, Marwan H. Elkassas* \\ Department of Cardiothoracic Surgery, Faculty of Medicine, Suez Canal University, Egypt
}

\begin{abstract}
Background: Pericardial effusion is commonly seen after coronary artery bypass surgery; large pericardial effusion develops at 4 to 10 days postoperatively in $30 \%$ of patients undergoing cardiac surgery. However, regional pericardial effusions are often localized posteriorly; one of the recent solutions to reduce this complication is posterior pericardiotomy, which also significantly reduces late pericardial effusion and late posterior tamponade. Aim: our target is to assess the impact and effectiveness of posterior pericardiotomy in reducing post-cardiac surgery pericardial effusion and tamponade. Patients and Methods: This prospective randomized case-controlled study was carried out in 64 patients. They were randomly assigned to one of 2 groups of 32 patients each. Longitudinal incision in the pericardium was made parallel and posterior to the left phrenic nerve in group one and not in group II, then we evaluated the results between the two groups as regards post operative pericardial effusion. Results: There were no statistically significant differences between the groups with respect to demographic or operative characteristics. The pericardiotomy group had lower incidence of postoperative pericardial effusion as $68.75 \%$ of the Pericardiotomy group had no effusion in the first seven days, while it was $43.3 \%$ in the control group, and none of the Pericardiotomy group developed severe pericardial effusion at any time. Conclusion: posterior pericardiotomy significantly reduce postoperative effusion and tamponade, and we recommend it in all the cases of cardiac surgery operations.
\end{abstract}

Keywords: tamponade, cardiac complications, massive effusion

\section{Introduction}

Pericardial effusion is commonly seen after coronary artery bypass surgery. It is usually small in amount and inconsequential. However, in some cases, pericardial effusion may be circumferential and quite large or it may be regional and loculated, either of which may impede cardiac filling, reduce cardiac output, and lead to tamponade ${ }^{(1)}$. Regional pericardial effusions are often localized posteriorly. Previous reports have dealt with the clinical features and management of this problem and have emphasized the high mortality rates associated with delayed treatment ${ }^{(2-5)}$. Large pericardial effusions develop at 4 to 10 days postoperatively in $30 \%$ of patients undergoing cardiac surgery. Large pericardial effusions are more common in patients with excessive early bleeding, after valve operations, and in cardiac transplant recipients ${ }^{(1,2)} \mathrm{Ef}$ fusions reach maximum size on the 10th day postoperatively in most cases, and generally regress spontaneously thereafter $^{(1)}$. Cardiac tamponade develops in only $1 \%$ of patients with pericardial effusion. Delayed tamponade may arise several days to weeks after the operation. Although delayed tamponade is infrequent, it is more 
common in patients treated with anticoagulants $^{(1)}$. Delayed moderate to massive pericardial effusion and low output syndrome with renal dysfunction is well known in cardiac surgery ${ }^{(1,3,4)}$.

After coronary operation, the space anterior to the heart may accommodate fluid and is easily drained from a chest drain; but behind the heart, adhesions between the inferior surface of the heart and the diaphragm may create an enclosed space ${ }^{(5)}$. Mulay and associates ${ }^{(6)}$ have demonstrated that posterior Pericardiotomy could drain freely into the left pleural space thereby reducing the prevalence of pericardial effusion. Late cardiac tamponade is a rare but serious complication, and it has been estimated to occur in up to $6 \%$ of patients after heart operations ${ }^{(7)}$. Significant delayed pericardial effusion occurred in $1.11 \%$, and $40 \%$ of these patients have posterior cardiac tamponade, Posterior pericardiotomy also significantly reduces late pericardial effusion and late posterior tamponade ${ }^{(8)}$. Posteriorpericardiotomy has recently been reported to reduce the prevalence of echocardiographically defined pericardial effusions from $40 \%$ in a control group to $8 \%$ in a pericardiotomy group ${ }^{(6)}$.

As this is an important issue in post cardiac surgery and as posterior pericardiotomy is one of its recent solutions, we conducted this prospective study to test the effectiveness of posterior pericardiotomy in reducing the postoperative pericardial effusion, and the development of late posterior cardiac effusions.

\section{Patients and Methods}

This prospective randomized case controlled study was carried out in 64 patients. They were randomly assigned to one of 2 groups of 32 patients each. They underwent coronary artery bypass grafting $(C A B G)$; we did not involved CABG with combined valve surgery; between December 2008 and May 2010 in Suez Canal University Hospital. Longitudinal incision in the pericardium was made parallel and posterior to the left phrenic nerve, extending from the left inferior pulmonary vein to the diaphragm (posterior pericardiotomy) in group I as described by Mulay and cowork$\mathrm{ers}^{(6)}$. Informed consent was obtained from all patients. Posterior pericardiotomy was not performed in group II.

Two chest tubes (one in the left pleural cavity and the other in anterior mediastinum) were inserted, and the pericardium was left open anteriorly in both groups. No drain was placed behind the heart to avoid tube-induced ventricular arrhythmias in both groups. Anesthetic medication and surgical techniques were similar in each group. Cardiopulmonary bypass was established with a roller pump non-pulsatile flow after anticoagulation with heparin (3 $\mathrm{mg} / \mathrm{kg}$ ), and activated clotting time was maintained for more than 450 seconds. Membrane oxygenators (Meados, Germany) were used in all cases. Heparin was reversed by protamine $(3.5 \mathrm{mg} / \mathrm{kg})$ at the end of the cardiopulmonary bypass. The left pleural cavity was opened in all patients, and patients with dense adhesion of left lung were excluded. All distal anastomosis was done in a single cross clamp period. The left internal thoracic artery (LITA) was used in all patients. Patients' demographics is summarized in Table (1). After routine closure of the chest, continuous suction (10-20 $\mathrm{mmHg}$ ) was applied to the drains, which were milked and stripped at 30minute intervals to ensure tube patency. Chest tubes were removed when the drainage was less than $20 \mathrm{ml} / \mathrm{h}$ for consecutive 4 hours. The presence of pericardial effusion was assessed by 2-dimensional echocardiography, which was performed in postoperative days 7 before discharge, 15 days, and one month after discharge by 
one experienced cardiologist. The presence of pericardial effusion on 2-dimensional echocardiography was assessed; the maximum diastolic separation between pericardium and epicardium was measured at the level of the tip of mitral valve leaflet, we defined mild effusion as pericardial effusion less than $1 \mathrm{~cm}$ not all around the heart, moderate as pericardial effusion all around the heart and more than $1 \mathrm{~cm}$ but less than $2 \mathrm{~cm}$, and severe as pericardial effusion more than $2 \mathrm{~cm}$ either localized or all around, cardiac tamponade was defined when any amount of effusion existed and was compressing the heart. Patients were examined echocardiographically after discharge. Patients with posterior effusion were recorded. Late posterior effusions and tamponade were recorded. In addition, results were expressed as mean \pm standard deviation. The Chi-squared test and Fischer's exact test were used to compare groups. SPSS software (SPSS, Inc., Chicago, IL, USA) was used for analyses. A $p$ value $<0.05$ was considered statistical significance.

\section{Results}

The differences between the 2 groups as regards to age, sex, hypertension, diabetes, number of the distal anastomosis, duration

Table 1: Demographic Data

\begin{tabular}{|l|lll|}
\hline Variables & Group I & Group II & $\mathrm{p}$ \\
\hline Mean Age (yrs) & $62.3 \pm 4.5$ & $63.2 \pm 3.5$ & 0.58 \\
Male gender (\%) & $63 \%$ & $59.37 \%$ & 0.39 \\
Diabetes (\%) & $40.62 \%$ & $43.75 \%$ & 0.5 \\
Hypertension (\%) & $56.25 \%$ & $53.12 \%$ & 0.68 \\
Smoker (\%) & $65.63 \%$ & $62.50 \%$ & 0.7 \\
Ejection Fraction (\%) & $50.12 \%$ & $48.90 \%$ & 0.8 \\
Aortic clamp time (min.) & 54.2 & 53.4 & 0.6 \\
Total bypass time (min.) & 62.5 & 64.1 & 0.46 \\
Graft per patient & 3.1 & 2.9 & 0.54 \\
\hline
\end{tabular}

of cross clamp, total bypass time, etc..., were not statistically significant (Table 1). As regards postoperative morbidity, myocardial infarction, renal impairment, stroke, ventilation time, and total hospital stay there were no statistically significant difference (Table 2)

Re-intubation was required because of respiratory insufficiency in two patients in group I and in 2 patients in group II. Sternal dehiscence was developed in one patient in each group. The only statistically significant difference between the two groups as regards post operative data was the incidence of post operative atrial fibrillation with trends to be lower in posterior pericardiotomy group ( $p$ value <0.005) (Table 2). As regards Echocardiography data and pericardial effusion, $68.75 \%$ of the Pericardiotomy group had no effusion in the first seven days, while it was $43.3 \%$ in the control group, and none of the Pericardiotomy group developed severe pericardial effusion at any time, while two patients $(6.25 \%)$ had severe pericardial effusion in the non-pericardiotomy group (Table 3). One case of tamponade (3.12\%) in the control group required reexploration, but no one of the Pericardiotomy group had tamponade (Table 3)

\section{Discussion}

A previous study by Kuralay and colleagues ${ }^{(10)}$ on the effects of posterior pericardiotomy on postoperative pericardial effusion showed a significant reduction in the incidence of postoperative pericardial effusion. Early and late effusions developed in $54 \%$ and $21 \%$ of patients without pericardiotomy respectively, and in none of those who had a posterior pericardiotomy. The incidence of delayed tamponade was also significantly lower in those who had a pericardiotomy (0\% vs. $10 \%$ ). 
Another study demonstrated the effectiveness of a posterior pericardiotomy in decreasing pericardial effusions after
$C A B G$, as well as reducing the incidence of supraventricular arrhythmias in the postoperative period $^{(6)}$.

Table 2: Preoperative morbidity factors in the studied groups

\begin{tabular}{|l|lll|}
\hline Morbidity & $\begin{array}{l}\text { Group I } \\
\text { (Total=32) }\end{array}$ & $\begin{array}{l}\text { Group II } \\
\text { (Total = 32) }\end{array}$ & P value \\
\hline $\begin{array}{l}\text { Transient Renal Impairment } \\
\text { (Serum Creatinine> } 1.5 \mathrm{mg} / \mathrm{dl} \text { ) }\end{array}$ & 3 & 4 & 0.93 \\
Stroke & 1 & 1 & 1 \\
Myocardial infarction & 2 & 1 & 0.8 \\
Mediastinitis & 1 & 1 & 1 \\
Atrial fibrillation & 6 & 13 & $0.004^{*}$ \\
Reoperation for bleeding & 1 & 2 & 0.85 \\
Hospital-acquired pneumonia & 2 & 2 & 1 \\
Ventilator time (h) & $7.9 \pm 6.3$ & $7.8 \pm 6$ & 0.9 \\
Intensive care unit stay (days) & $1.3 \pm 0.7$ & $1.2 \pm 0.5$ & 0.8 \\
Hospital stay (days) & $7.9 \pm 4.7$ & $8.5 \pm 5.1$ & 0.9 \\
\hline *statistically significant & & & \\
\hline
\end{tabular}

Table 3: Postoperative ECHO data (Pericardial Effusion)

\begin{tabular}{|l|lllll|}
\hline Time & \multicolumn{5}{|c|}{ Amount } \\
\hline Group I & None & Mild & Moderate & Severe & Tamponade \\
Seven days & $22(68.75 \%)$ & $6(18.75 \%)$ & $4(12.5 \%)$ & 0 & 0 \\
15 Days & $25(78.12 \%)$ & $4(12.5 \%)$ & $3(9.37 \%)$ & 0 & 0 \\
30 Days & $29(90.62 \%)$ & $2(6.25 \%)$ & $1(3.25 \%)$ & 0 & 0 \\
Group II & & & & & \\
Seven Days & $13(40.3 \%)$ & $11(34.37 \%)$ & $5(15.62 \%)$ & $2(6.25 \%)$ & $1(3.12 \%)$ \\
15 Days & $14(43.75 \%)$ & $13(40.62 \%)$ & $4(12.5 \%)$ & $1(3.25 \%)$ & 0 \\
30 Days & $20(62.5 \%)$ & $9(28.12 \%)$ & $3(9.37 \%)$ & 0 & 0 \\
\hline
\end{tabular}

Group I=Pericardiotomy; group II= control

Asimakopoulos and coworkers ${ }^{(11)}$ have shown that posterior pericardiotomy was more effective for pericardial drainage, and the incidence of post operative pericardial effusion is significantly lower in this group. The results of our study also revealed highly significant differences between the two groups, indicating that a posterior pericardiotomy after coronary artery bypass graft was very effective in reducing the incidence and severity of postoperative pericardial effusion, and the adverse consequences of delayed tamponade were thereby eliminated. We also can noticed that our incidence of pericardial effusion is higher, but we can explain that as we did not used a well calibrated suction system on our drainage tubes as most of the other centers. After coronary operation, the space anterior to the heart may accommodate fluid and is easily drained from a chest drain; but behind the heart, adhesions between the inferior surface of the heart and the diaphragm may create an enclosed space. Mulay and associates ${ }^{(6)}$ have demonstrated that posterior pericardiotomy could drain freely into the left pleural space thereby reducing the prevalence of 
pericardial effusion. We performed posterior pericardiotomy posterior and parallel to phrenic nerve from the left inferior pulmonary vein toward the inferior vena cava and diaphragm. This area of the pericardial cavity was chosen because it was expected to be the most effective in preventing the accumulation of fluid postoperatively.

\section{Conclusions}

We conclude that posterior pericardiotomy is technically simple as well as safe and effective. Therefore, it is recommended in coronary artery bypass surgery to reduce the incidence of postoperative pericardial effusion.

\section{References}

1. Angelini GD, Penny WJ, el-Ghamary F, West RR, Butchart EG, Armistead SH, Breckenridge IM, Henderson $\mathrm{AH}$. The incidence and significance of early pericardial effusion after open-heart surgery. Eur J Cardiothorac Surg 1987;1(3):165-168.

2. Ikaheimo MJ, Huikuri HV, Airaksinen J, Korhonem U, Linnaluoto MK, Tarkka MR, Takkunen JT. Pericardial effusion after cardiac surgery: incidence, relation to the type of surgery, antithrombotic therapy, and early coronary bypass graft patency. Am Heart J 1988;116(1 pt1):97-102.

3. Chuttani K, Pandian NG, Mohanty PK, Rosenfield K, Schwartz SL, Udelson JE, Simonetti J, Kusay BS, Caldeira ME. Left ventricular diastolic collapse. An echocardiographic sign of regional cardiac tamponade. Circulation 1991;83(6):1999-2006.

4. Borkon AM, Schaff HV, Gardner TJ, Merrill WH, Brawley RK, Donahoo JS, Watkins L Jr,
Weiss JL, Gott VL. Diagnosis and management of postoperative pericardial effusions and late cardiac tamponade following openheart surgery. Ann Thorac Surg 1981; 31(6):512-519.

5. Yilmaz AT, Arslan M, Demirkliç U, Kuralay E, Ozal E, Bingöl H, Oz BS, Tatar H, Oztürk OY. Late posterior cardiac tamponade after open heart surgery. J Cardiovasc Surg 1996; 37(6):615-620.

6. Mulay A, Kirk AJ, Angelini GD, Wishheart JD, Hutter JA. Posterior pericardiotomy reduces the incidence of supra-ventricular arrhythmias following coronary artery bypass surgery. Eur J Cardiothorac Surg 1995;9(3):150152.

7. Chuttani K, Tischer MD, Pandian NG, Lee RT, Mohanty PK. Diagnosis of cardiac tamponade after cardiac surgery: relative value of clinical, echocardiographic and hemodynamic signs. Am Heart J 1994;127(4 pt 1):913918.

8. Sahni J, Ivert T, Herzfeld I, Brodin LA. Late cardiac tamponade after open heart surgery. Scand J Thorac Cardiovasc Surg 1991; 25(1):63-68.

9. D'Cruz IA, Overton DH, Pai GM.Pericardial complications of cardiac surgery: emphasis on the diagnostic role of echocardiography. J Card Surg 1992;7(3):257-258.

10. Kuralay E, Ozal E, Demirkili U, Tatar H. Effect of posterior pericardiotomy on postoperative supraventricular arrhythmias and late pericardial effusion (posterior pericardiotomy). J Thorac Cardiovasc Surg 1999; 118(3):492-495.

11. Asimakopoulos G, Della-Santa R, Taggart DP. Effects of posterior pericardiotomy on the incidence of atrial fibrillation and chest drainage after coronary revascularization: a prospective randomized trial. J Thorac Cardiovasc Surg 1997;113(4):797-799. 